December 1st, 12pm EST

\section{THE ULTIMATE VIRTUAL SEMINAR SERIES IN GENE} EXPRESSION STUDIES
GENE EXPRESSION UNIVERSITY

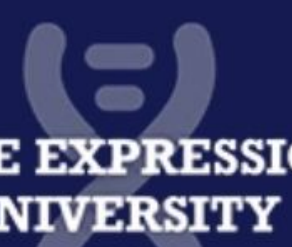

Register Now $>>$

\section{applied biosystems}

by Thermo Fisher Scientific

\section{ThermoFisher \\ S C I EN T I F I C}

WILEY 


\title{
Spectrum of pathogenic variants and founder effects in amelogenesis imperfecta associated with MMP20
}

\author{
Georgios Nikolopoulos ${ }^{1,2}$ (1) | Claire E. L. Smith ${ }^{1}$ (1) | James A. Poulter ${ }^{1}$ (이 | \\ Gina Murillo $^{3}$ (1) | Sandra Silva ${ }^{4}$ | Teresa Lamb ${ }^{5}$ | Ian R. Berry ${ }^{6}$ | \\ Catriona J. Brown ${ }^{7}$ | Peter F. Day ${ }^{8,9}$ (1) | Francesca Soldani ${ }^{9}$ | \\ Suhaila Al-Bahlani $^{10}$ | Sarah A. Harris ${ }^{11,12}$ @ | Mary J. O'Connell ${ }^{13,14}$ ( ) | \\ Chris F. Inglehearn ${ }^{1}$ () | Alan J. Mighell ${ }^{2}$ (b)
}

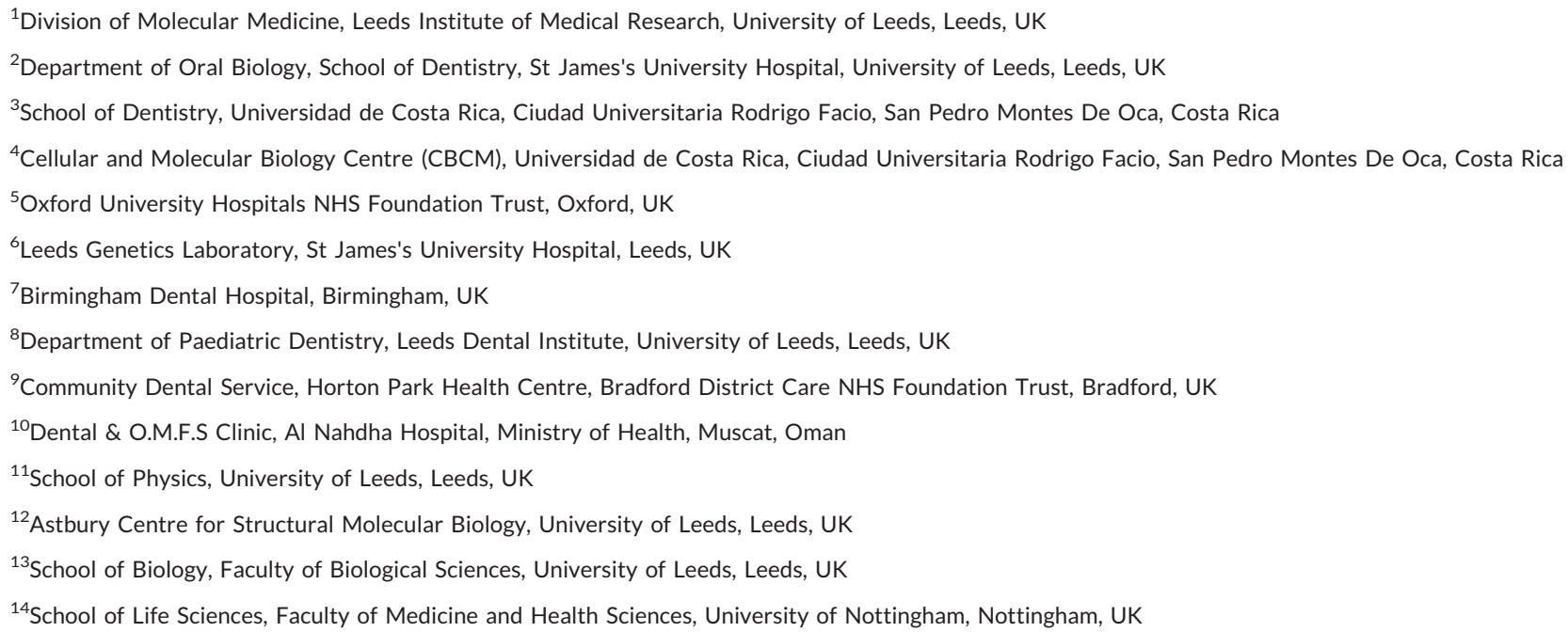

\section{Correspondence}

Alan J. Mighell, Department of Oral Biology, School of Dentistry, James's University Hospital, University of Leeds, Worsley Bldg, Leeds LS2 9LU, UK.

Email: A.J.Mighell@leeds.ac.uk

\begin{abstract}
Amelogenesis imperfecta (AI) describes a heterogeneous group of developmental enamel defects that typically have Mendelian inheritance. Exome sequencing of 10 families with recessive hypomaturation Al revealed four novel and one known variants in the matrix metallopeptidase 20 (MMP20) gene that were predicted to be pathogenic. MMP20 encodes a protease that cleaves the developing extracellular enamel matrix and is necessary for normal enamel crystal growth during amelogenesis. New homozygous missense changes were shared between four families of Pakistani heritage (c.625G $>$ C; p.(Glu209GIn)) and two of Omani origin (c.710C>A; p.(Ser237Tyr)). In two families of UK
\end{abstract}

Georgios Nikolopoulos and Claire E. L. Smith contributed equally to this study. 
origin and one from Costa Rica, affected individuals were homozygous for the previously reported c.954-2A>T; p.(Ile319Phefs*19) variant. For each of these variants, microsatellite haplotypes appeared to exclude a recent founder effect, but elements of haplotype were conserved, suggesting more distant founding ancestors. New compound heterozygous changes were identified in one family of the European heritage: c.809_811+12delinsCCAG; p.(?) and c.1122A>C; p.(GIn374His). This report further elucidates the mutation spectrum of MMP2O and the probable impact on protein function, confirms a consistent hypomaturation phenotype and shows that mutations in MMP2O are a common cause of autosomal recessive $\mathrm{Al}$ in some communities.

\section{KEYWORDS}

amelogenesis imperfecta, enamel, founder effect, hypomaturation AI, MMP20

\section{1 | INTRODUCTION}

The hardness of dental enamel is remarkable and is a result of its high mineral and low protein content (C. E. Smith, 1998). The process of enamel formation, amelogenesis, begins with the production of a secreted soft enamel protein matrix, which is then transformed into a mature, organized structure of rod and interrod enamel crystallites almost devoid of protein. Crucial to this transformation process is the secretory stage enamel protease, matrix metallopeptidase 20 (MMP20) [NM_004771.4, ENST00000260228.2] (MIM \#604629).

MMP2O is a zinc-dependent endopeptidase that is secreted in trace amounts during the secretory and transition stages of amelogenesis by ameloblasts (Llano et al., 1997; Seymen et al., 2015). Once activated, it selectively cleaves the secreted enamel proteins: amelogenin, enamelin, and ameloblastin into several products with distinct functional roles (Simmer \& Hu, 2002). MMP20 is also thought to facilitate ameloblast movement during secretion, through cell-cell communication, and may influence ameloblast gene expression (Guan \& Bartlett, 2013). These activities create a newly voided space within which enamel crystallites are able to grow in width and thickness, and finally, to interlock (Bartlett, 2013). If they are not removed, the enamel proteins occupy the enamel volume and restrict the growth of the enamel crystallites. This produces immature enamel that fails prematurely due to its inability to resist the mechanical stress resulting from biting and chewing.

Mutations in MMP20 and 19 other genes have been shown to cause nonsyndromic amelogenesis imperfecta (Al) with autosomal dominant, autosomal recessive, or X-linked inheritance (J. W. Kim et al., 2019; C. E. L. Smith et al., 2017, 2020; and http://dna2.leeds.ac.uk/LOVD/genes), while perhaps as many again have been implicated in syndromic $\mathrm{Al}$ (Dubail et al., 2018; Wright et al., 2015). Some of these genes encode proteins processed by MMP20, such as the enamel matrix proteins AMELX (MIM \#300391), AMBN (MIM \#610259), and ENAM (MIM \#606585). Other genes implicated in Al include the second enamel matrix protease, KLK4 (MIM \#603767), and cell adhesion proteins, such as LAMA3 (MIM \#600805), LAMB3 (MIM \#150310), and COL17A1 (MIM $\# 113811$ ). Al, therefore, describes a heterogeneous group of conditions characterized by inherited enamel defects in both dentitions. It can present as a hypoplastic phenotype, where a deficit in secretion results in the formation of a reduced volume of mineralized enamel, or as hypomineralized $\mathrm{Al}$, whereby a failure in maturation results in the enamel of full thickness but which is soft or brittle and fails prematurely (Gadhia et al., 2012). Hypomineralized Al can be further subclassified as hypomaturation, caused by incomplete removal of protein from the developing enamel, or hypocalcification, caused by insufficient transport of calcium ions into the forming enamel (Smith et al., 2017), though these phenotypes often overlap. Mutations in MMP20 cause autosomal recessive hypomaturation Al. Fourteen pathogenic variants have been reported to date (Table S1), most of which are located in the catalytic domain of MMP2O and are thought to affect the stability and functionality of the protein structure.

This study describes the identification of pathogenic MMP2O variants in 10 families segregating autosomal recessive hypomaturation $\mathrm{Al}$, providing additional insights into the spectrum of MMP20 mutations and associated phenotype. Four novel variants are reported, two of which are relatively common founder mutations in specific populations. Additionally, we perform molecular dynamics (MD) simulations of variants in the catalytic domain of MMP20 to examine the predicted effect that these changes have on the protein. This study increases the total reported pathogenic MMP20 variants to 17 , suggests that defects in MMP20 are a more common cause of Al than was previously reported and enriches our understanding of the effects that mutations in the catalytic domain of MMP2O can have on its functionality.

\section{2 | MATERIALS AND METHODS}

\section{1 | Patients}

Individuals from each of the 10 families were recruited following informed consent in accordance with the principles outlined in the Declaration of Helsinki, with local ethical approval (REC 13/YH/0028). These 10 families are part of a larger Al cohort presenting with a variety of $\mathrm{Al}$ phenotypes. A diagnosis of $\mathrm{Al}$ was made by a dentist after clinical 
examination, based on the physical appearance of the dentition, and was confirmed by dental X-ray. Genomic DNA was obtained via venous blood samples, using conventional extraction techniques, or from saliva using Oragene ${ }^{\circledR}$ DNA Sample Collection kits (DNA Genotek) and extracted according to the manufacturer's instructions.

\subsection{Whole-exome sequencing (WES) and analysis}

Three micrograms of genomic DNA from a single individual from each family (marked with an arrow on the pedigrees shown in
Figure 1) were subjected to WES and analyzed as described previously (C. E. L. Smith et al., 2019). Variants present in the dbSNP150 database of NCBI or the Genome Aggregation Database (gnomAD; http://gnomad.broadinstitute.org) with a minor allele frequency $\geq 1 \%$ were excluded. The potential pathogenic effect of the variants was predicted using Combined AnnotationDependent Depletion (CADD v1.3; https://cadd.gs.washington. edu) (Rentzsch et al., 2019), the Sorting Intolerant From Tolerant algorithm (SIFT; Sim et al., 2012), Protein Variation Effect Analyzer(PROVEAN; Choi et al., 2012), and MutPred2 (http://mutpred. mutdb.org/index.html). The potential effect on splicing for each

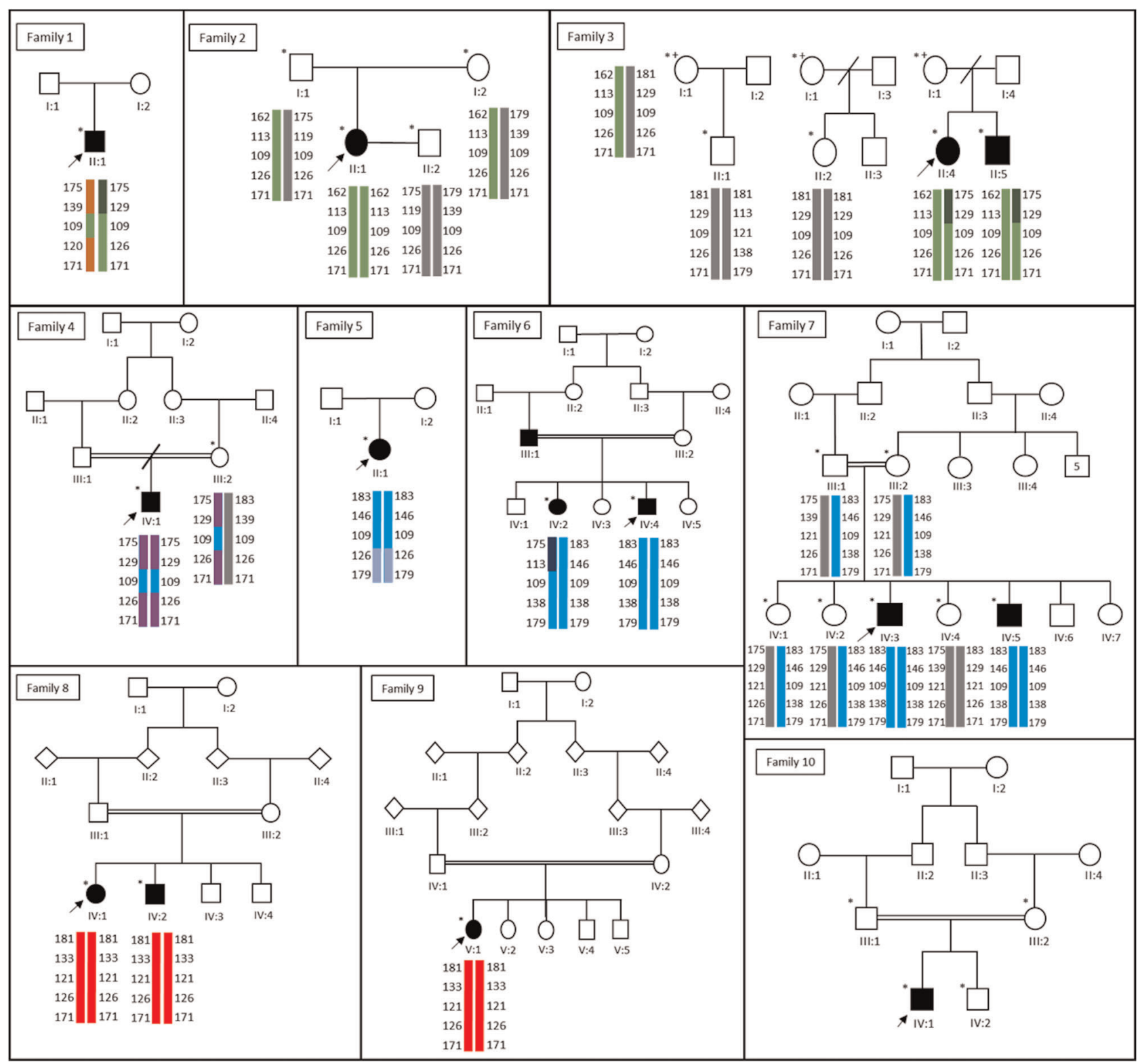

FIGURE 1 Pedigrees of the 10 families recruited for this study. The genotypes determined from the microsatellite markers are presented beneath each individual examined. Families 1, 2, and 3 carry the c.954-2A $>$ T variant; Families 4, 5, 6, and 7 carry the c.625G $>C$ variant; Families 8 and 9 carry the c.710C>A variant; and Family 10 has the c.809_811+12delinsCCAG and c.1122A>C variants. The common haplotypes are presented in the same color and the haplotypes without a pathogenic variant are colored gray. The markers are presented in the order: 11cen, D11S940, D11S1339, MMP20, D11S4108, D11S4159, D11S4161, 11qter. The recruited family members are marked with an asterisk and the proband in each family is indicated with a black arrow 
intronic variant was predicted by the Human Splicing Finder v3.1 (http://umd.be/HSF3/HSF.shtml).

\section{3 | Polymerase chain reaction (PCR) and Sanger sequencing}

Mutations were confirmed and segregation was performed for all available family members, marked with $\left(^{*}\right)$ on each pedigree of Figure 1. Primer sequences can be found in Table S2. Sanger sequencing was performed using the BigDye Terminator v3.1 kit (Life Technologies) according to the manufacturer's instructions and resolved on an $\mathrm{ABI} 3130 \mathrm{xl}$ sequencer (Life Technologies). Results were analyzed using SeqScape v2.5 (Life Technologies).

\section{4 | Microsatellite analysis}

Primer sequences for markers were obtained from the UCSC genome browser and standard HEX-tagged primers (Sigma-Aldrich; Table S2) were used to assess the flanking haplotypes of MMP2O. The analysis was performed as described previously (Nikolopoulos et al., 2020).

\section{5 | Protein structure analysis}

The tertiary structure of MMP20 has been determined in atomistic detail by nuclear magnetic resonance (NMR) (PDB: 2JSD) (Arendt et al., 2007), which provides the starting structure for MD simulations. Amino acid substitutions were made in the wild type (WT) structure using the Chimera visualization tool (Pettersen et al., 2004). MD simulations were performed using AMBER18 (Case et al., 2018). Protocols used to perform the MD simulations are in the Supporting Information Methods. Rhapsody was used for in silico saturation mutagenesis analysis (Ponzoni et al., 2020) and the prediction of the changes in solvent accessibility, residue occlusion, and free energy $(\Delta \Delta G)$ of the mutated proteins was performed with Site-Directed Mutator (SDM) (Pandurangan et al., 2017).

\section{3 | RESULTS}

Ten unrelated families presenting with features consistent with autosomal recessive hypomaturation $\mathrm{Al}$, in the absence of any cosegregating disease, were recruited for the study (Figures 1 and 2). Genomic DNA from affected members of each family was subjected to exome sequencing. Detailed coverage statistics can be found in
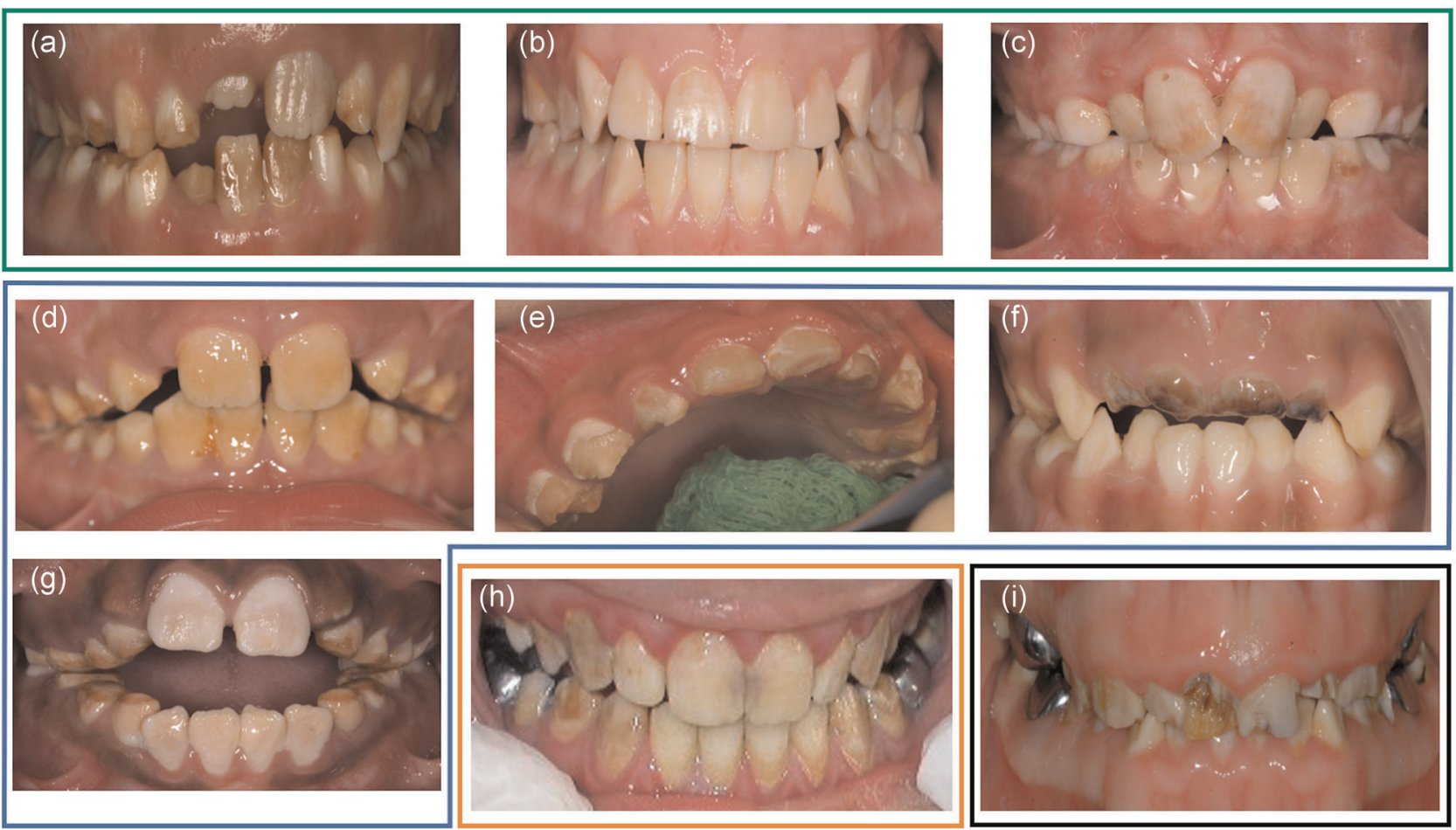

FIGURE 2 Clinical images for nine probands at different chronological ages, illustrating the spectrum of variations that can be observed within the overall, typical features of hypomaturation amelogenesis imperfecta that have been subjected to different post-eruptive environments. (a-c) Families 1-3, who all have European heritage and share the same homozygous MMP20 c.954-2A>T; p.(Ile319Phefs*19) variant. (d-g) Families 4-7, who all have Pakistani heritage and share the same homozygous MMP20 c.625G>C; p.(Glu209Gln) variant. (h) Family 9 who, along with Family 8 (no clinical images available), has Omani heritage, and both have the same homozygous MMP20 c.710C>A; p.(Ser237Tyr) variant. (i) Family 10, of European heritage, which was found to segregate the compound heterozygous MMP20 c.809_811+12delinsCCAG; p.(?) and c.1122A>C; p.(GIn374His) variants 
Table S3. Samples were sequenced in different batches over a period of some years, with considerable variation in coverage between batches. Variant files were filtered to select rare variants with high predicted pathogenicity, then variants in known Al-causing genes were highlighted. This revealed biallelic mutations in MMP2O (Table S4) in each family. The position of these variants in the gene is shown in Figure 3, along with representative electropherograms for each novel variant. PCR and Sanger sequencing confirmed the variants segregated with $\mathrm{Al}$ in all available family members.

In Families 1, 2, and 3, a homozygous frameshift variant (NM_004771: c.954-2A>T, NP_004762: p.(lle319Phefs*19)) was identified in intron 6. This has been published previously as a cause of autosomal recessive hypomaturation $\mathrm{Al}$ and is expected to lead to retention of the sixth intron (J. W. Kim et al., 2005). To confirm this hypothesis, we attempted to perform reverse-anscriptase PCR of the MMP20 transcript on control blood complementary DNA (cDNA). However, no amplification was achieved, suggesting that the level of MMP20 expression in blood is below the threshold for detection.

A novel homozygous missense mutation, c.625G $>C$, p.(Glu209GIn), was identified in exon 4 as the cause of disease in Families 4, 5, 6, and 7. This is known variant rs199788797, which has not previously been associated with a disease phenotype. In the gnomAD database, this variant has a frequency of 0.000457 in the South Asian population but is absent from all other reported populations. E209 is fully conserved in the mammalian clade, as shown in Figure S1, using the sequences listed in Table S5. Additionally, the mutation is predicted to be damaging (Table S6).

In Families 8 and 9, a novel homozygous missense mutation, c.710C >A, p.(Ser237Tyr) was identified in exon 5. This variant is absent from databases, is evolutionarily conserved in all the mammalian species analyzed (Figure S1) and is predicted to be damaging by all algorithms used (Table S6).
The affected individual in Family 10 was found to be a compound heterozygote for a novel missense mutation c.1122A $>C$, p.(GIn374His) in exon 8 and a novel deletion-insertion (delins) variant: c.809_811+12delinsCCAG, p(?), spanning the splice donor site of intron 5. Both are absent from variant databases. Variant p.(Gln374His) is predicted to be damaging (Table S6), and Q374 is conserved in the mammalian clade (Figure $\mathrm{S1}$ ). Interestingly, mouse Mmp20 has a histidine in the equivalent position. This presumably does not have a detrimental effect on the function of the mouse protein, perhaps due to other changes of the mouse gene that balance the protein or reflecting differences in enamel development and function between mouse teeth, which grow throughout life, and human teeth which do not. Nevertheless, mutation prediction software does classify this variant as pathogenic; it is absent from the gnomAD database, and when paired with a second MMP20 variant it has given rise to a hypomaturation phenotype consistent with biallelic MMP2O disease. The delins variant is predicted by Human Splicing Finder to disrupt the intron 5 splice donor site, possibly leading to retention of the fifth intron (Figure S2).

The families that share variants (Families 1, 2, and 3 with c.954-2A >T, Families 4-7 with c.625G >C, and Families 8 and 9 with c. $710 \mathrm{C}>\mathrm{A}$ ) also originate from the same ethnic backgrounds. To determine whether these families share common founder haplotypes at the MMP20 locus, we genotyped five microsatellite markers across a $1.5 \mathrm{cM} / 3 \mathrm{Mb}$ region of chromosome $11 \mathrm{q} 22$ in each family, in the order: 11cen, D11S940, D11S1339, MMP20, D11S4108, D11S4159, D11S4161, 11qter. The haplotype analysis is shown in Figure 1.

In Omani families 8 and 9, carrying the c.710C >A variant, haplotyping suggests they are closely related. However, the picture is more complex in the remaining two family groups. Families 1-3 (the first from Costa Rica, the remaining two from the United Kingdom) carry the previously published c.954-2A>T variant. The proband in

(a)

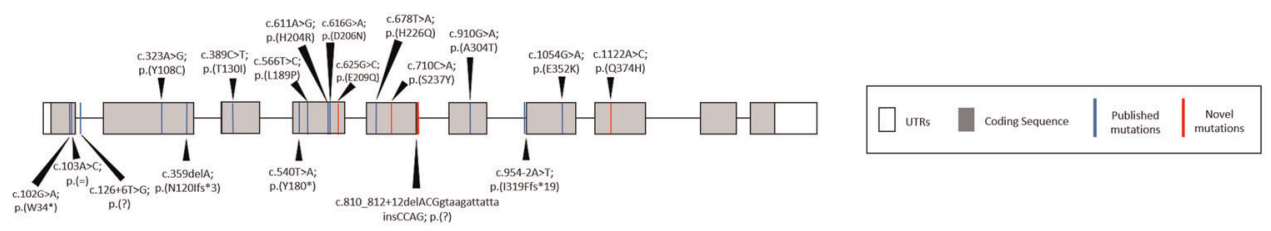

(b)
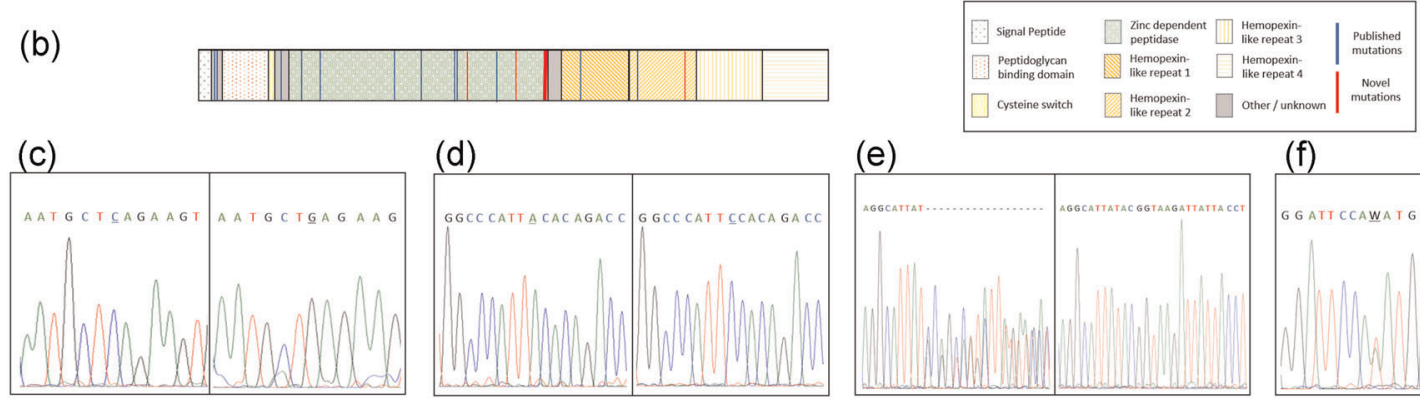

(d)

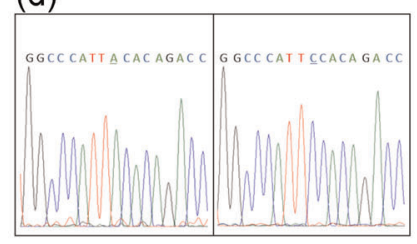

(e)

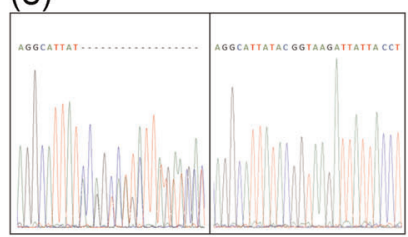

(f)

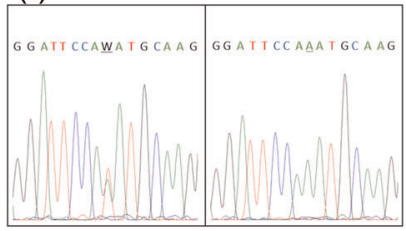

FIGURE 3 (a) MMP20 gene diagram. The positions of all known mutations are marked on the gene with a vertical bar, while novel variants are highlighted in red. (b) Representation of the protein domains with all known mutations marked with vertical bars, as in (a). The zincdependent peptidase domain is the catalytic domain of the protein, where most pathogenic variants have been found. (c-f) $A$ representative electropherogram of each of the novel variants found in this study, (c) c.625G>C variant and wild type (WT), (d) c.710C>A variant and WT, (e) c.809_811+12delinsCCAG variant and WT, (f) c.1122A>C variant and WT 
Family 2 is homozygous for a haplotype also seen in Family 3, but the second haplotype in Family 3 has proximal recombination. Family 1 could share the recombinant haplotype observed in Family 3 together with an unrelated haplotype, but without phase information this cannot be confirmed. Interestingly, all three families are homozygous for D11S4108 $100 \mathrm{~kb}$ from MMP20. Families 4-7, of UK-Pakistani origin, carry the $\mathrm{c} .625 \mathrm{G}>\mathrm{C}$ variant. The proband in Family 6 is homozygous for the same haplotype segregating in Family 7. However, a second haplotype with distal recombination is seen in the second affected sibling in Family 6, suggesting the affected (unsampled) father carries both haplotypes. Family 5 is homozygous for a third haplotype, again identical at the proximal end to that in Family 7 but recombinant at the distal end. In contrast, Family 4 is homozygous for a fourth haplotype identical to the Family 7 haplotype at the distal end but proximally recombinant. Again, all four families are homozygous for the immediately adjacent marker D11S4108.

The catalytic center of MMP20 is a 160-residue domain containing the zinc-dependent peptidase active site. It contains one catalytic and one structural zinc ion; the structures modeled here additionally contain two calcium ions, which are also structural. We assessed how missense variants affect the protein function by performing MD simulations of the relevant mutated proteins. In addition, to demonstrate the key role of metal ions in maintaining MMP20 structure, we performed MD simulations in which the metal ions were removed. We compared the protein structures and dynamics observed for the WT with metal ions present, with those obtained for p.(Glu209GIn) and p.(Ser237Tyr) from this study (see Figure 4a) and p.(Thr130lle) and p.(Leu189Pro) from the literature (Figure 4b); and also with results of the WT in the absence of metal ions. Figure $4 c$ shows the root mean squared deviation (RMSD) of each of the simulations from their starting structures. The averages calculated for the last 300 ns of each repeat are in Table S7, and the repeats for the entire 900-ns simulation are shown separately in Figure S3. The calculated RMSD values are continuously adjusted and recalculated during the $900 \mathrm{~ns}$ of the simulation. As such, the final $300 \mathrm{~ns}$, during which the plots have stabilized, are selected as the representative RMSD values for each simulation, and so the average values presented in Table S7 are used for the comparison among the variants. An increase in RMSD relative to the WT, implying decreased protein stability, was observed for all variants, apart from p.(Thr130lle). We also analyzed the changes in three key interatomic interactions between the WT and the variants, atomic fluctuations (Figure S4a), hydrogen bonding interactions (Table S8), and salt bridges (Table S9), to provide insight into why these particular variants cause functionally deleterious changes in protein structure. The most significant structural distortions were observed in the simulations performed in the absence of structural zinc and calcium ions (see Figure S4b,c).

In silico saturation mutagenesis of the MMP20 active site performed by Rhapsody shows that there are regions of the protein that are significantly more likely to cause a pathogenic effect when the residues located there are altered (Figure S5). These regions largely correlate with the sites of the known and novel variants and have an increased PolyPhen-2 score (Figure S5). The Rhapsody analysis was limited to the catalytic domain of MMP2O because it relies on the availability of a tertiary structure. The results of the SDM analysis are presented in Table S10, showing the changes of free energy, residue occlusion, and solvent accessibility for the WT and each mutant, respectively.

\section{4 | DISCUSSION}

Exome sequencing in a cohort of nonsyndromic Al families revealed biallelic MMP20 variants in 10 autosomal recessive families. MMP20 is a protease that plays an essential role during the secretory and early transition stages of amelogenesis. Defects in MMP20 cause Al in both humans and mice, due to a failure to process, degrade, and remove proteins from the extracellular matrix scaffold upon which the developing dental enamel is formed. The hypomaturation phenotype observed in these families fits with this hypothesis, with the dental enamel being of normal volume but characterized by a loss of translucency, discoloration, and premature enamel loss. Posteruptive changes probably determine the pattern of premature enamel failure. The phenotypes observed in the families presented are consistent with those described in previous reports of $\mathrm{Al}$ due to MMP20 variants.

The variant identified in Families 1-3, (c.954-2A>T), has been reported previously (Gasse et al., 2017; J. W. Kim et al., 2005; Prasad et al., 2015; Wright et al., 2011), but it has not been possible to confirm the effect on splicing experimentally since MMP2O is not expressed in blood. The prediction that the mutation most likely results in the loss of exon 7 from the MMP20 transcript (J. W. Kim et al., 2005), therefore, remains speculative. If confirmed, this would break the reading frame and lead to an abnormal transcript, which would be expected to be subject to nonsense-mediated decay. MMP20 cDNA cannot be obtained from the blood due to undetectable expression levels; however, it could possibly be obtained from tissues, such as tonsil or appendix, as expression has been detected there and these are routinely removed through routine surgery. However, altered splicing in these tissues would only suggest that splicing is altered in ameloblasts, instead CRISPR-Cas9 editing of this gene within ameloblast cell lines might be a better model to determine whether splicing is affected.

The missense variant identified in Families 4-7, p.(Glu209GIn), is novel and replaces a negatively charged glutamic acid residue with neutral glutamine. E209 is a coordinating ligand for calcium ion binding and is, therefore, critical to the function of MMP20 (Andreini et al., 2004; Arendt et al., 2007; Yamakoshi et al., 2013). Replacement with glutamine decreases protein stability relative to WT in the MD simulations (see Table S7). Inspection of the WT atomistic structure shows that E209 has a pair of carboxylate oxygen atoms oriented toward the nearby $\mathrm{Ca}^{2+}$ ion. In E209, the average distance is <4 A for both MD repeats (Table S9). However, the mutant Q209 only has one oxygen; in one of the two duplicate MD trajectories, the 


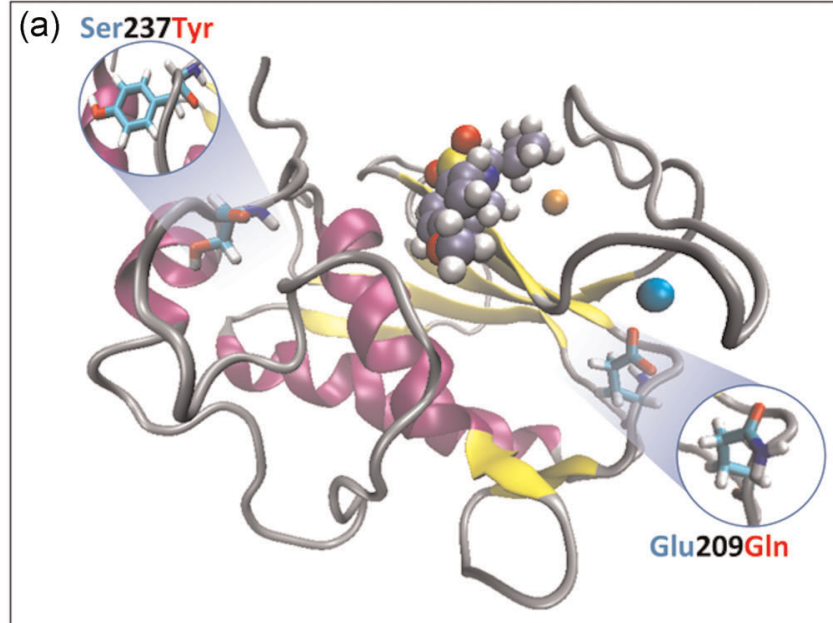

(b)

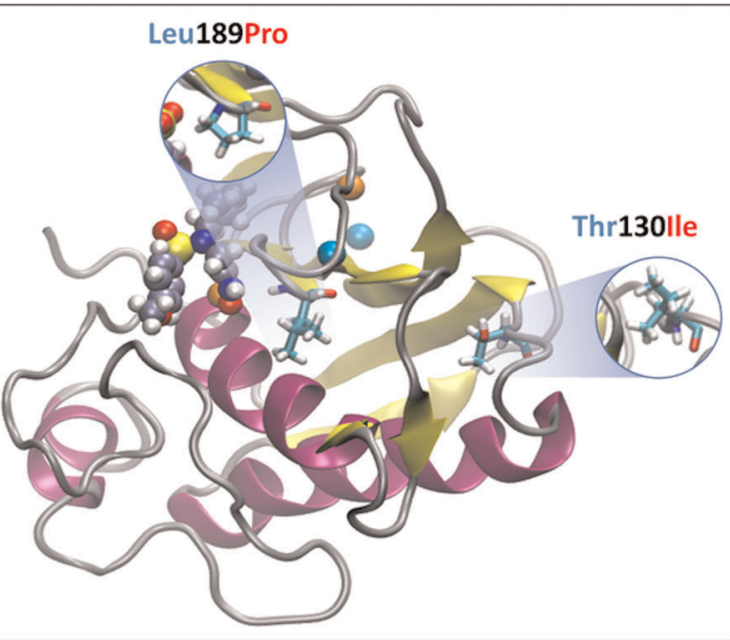

(c)

RMSD

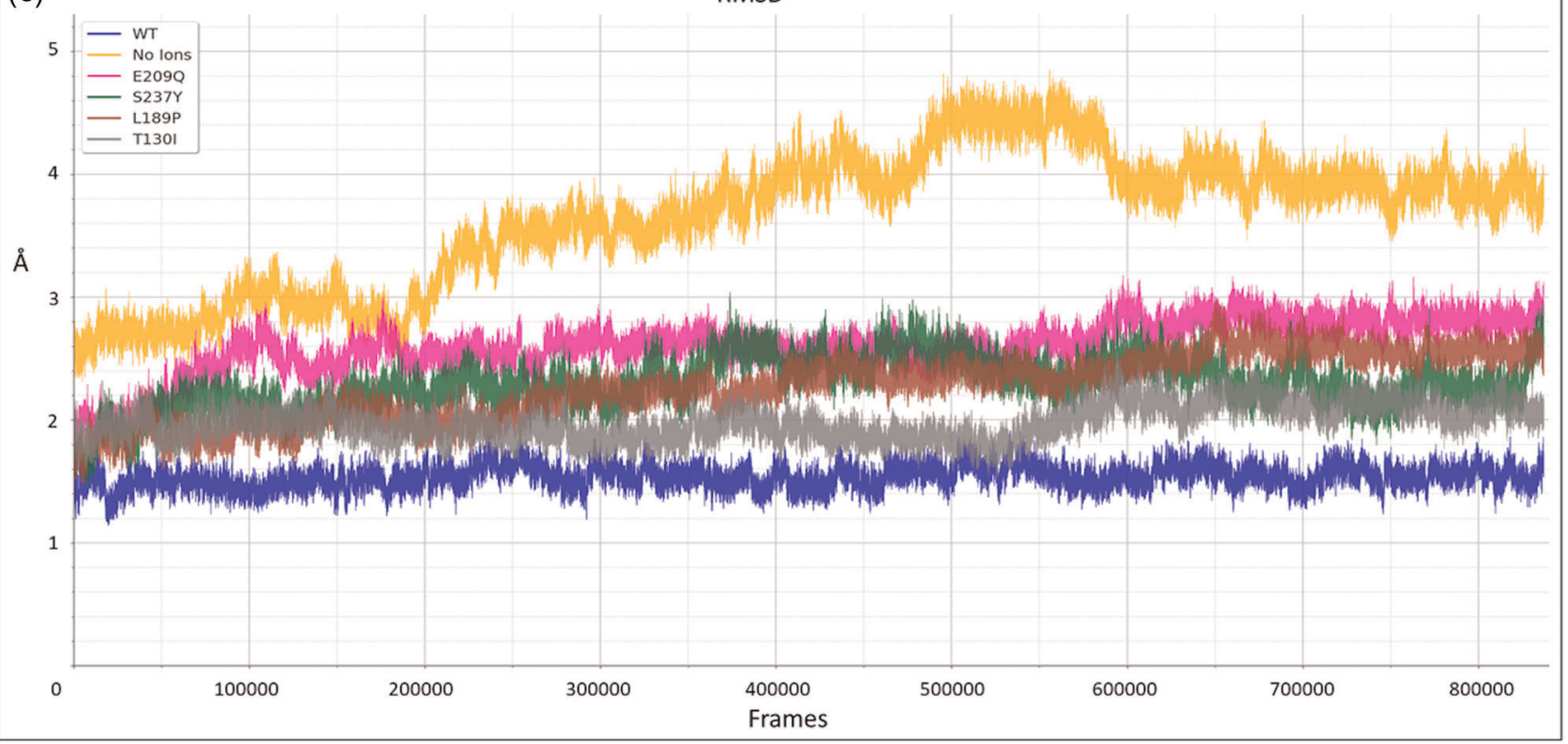

FIGURE 4 The tertiary structure of the catalytic domain of MMP20, based on the PDB: 2JSD nuclear magnetic resonance model. (a) The wild-type (WT) protein structure with the two novel variants, c.625G>C; p.(Glu209GIn) of Families 4-7 and c.710C>A; p.(Ser237Tyr) of Families 8 and 9, of the active site are presented in the inlays. (b) The WT protein structure with the inlays showing two of the known variants of the active site of MMP20, c.389C>T; p.(Thr130lle), and c.566T>C; p.(Leu189Pro). (c) The root mean square deviation (RMSD) of each modeled variant during molecular dynamics simulations of $900 \mathrm{~ns}$. An increase of RMSD value corresponds to a loss of stability, with the WT MMP20 structure being the most stable and the MMP20 structure in the absence of structural zinc and calcium ions being the least stable

interaction between this amide oxygen and $\mathrm{Ca}^{2+}$ remains strong (an interatomic distance of $2.5 \AA$ ) but in the other it is completely disrupted (interatomic distance of $6.9 \AA$ ), allowing it to form non-native hydrogen bonds with other residues (see Table S8).

The novel missense variant identified in Families 8 and 9 replaces a small serine residue with a larger aromatic tyrosine, p.(Ser237Tyr) in a highly conserved part of the catalytic domain of MMP20. Correspondingly, the SDM analysis identifies an increase in the folding free energy of the protein relative to the WT (Table S10), implying that the variant is less stable. Moreover, in the MD simulations, the larger and more hydrophobic Y237 results in an increased RMSD (2.35 $\mathrm{A})$ relative to the WT $(1.55 \AA)$ ). In these trajectories, the main backbone hydrogen bond formed by $\mathrm{S} 237$ (with M244) is also formed by Y237. However, the number of side-chain hydrogen bonds is reduced in Y237 compared with S237 (see Table S8) because the corresponding tyrosine oxygen protrudes too far into the solvent to participate in interatomic interactions within the protein.

In Family 10, the delins variant disrupts the intron $5^{\prime}$ splice donor site and is therefore again likely to lead to a transcript that is subject to nonsense-mediated decay. The novel c.1122A $>C$, p.(GIn374His) variant changes a hydrophilic glutamine residue for a hydrophobic histidine in the C-terminal hemopexin-like domain, as shown in Figure 3a, disrupting the folding of the protein and potentially leading to a loss of function. 
Microsatellites were used to determine whether the variants shared in each of the three family groups were founder alleles, implying these families are related rather than that these sites are mutation hotspots. Family 1 is from Costa Rica, the population of which is largely of mixed European and indigenous American descent. Families 2 and 3 are Caucasian European families from the United Kingdom. The c.954-2A>T variant has been reported previously in at least six families from France (Gasse et al., 2017; Prasad et al., 2015) and one from the United States (J. W. Kim et al., 2005). Ethnicity is given as Caucasian by Prasad and co-workers but is not given in the remaining reports. Our haplotype analysis shows that this variant is present on three different chromosomal backgrounds, but elements of the haplotype, in particular the genotype of D11S4108 $100 \mathrm{~kb}$ from MMP20, appear conserved between families. This suggests they may be related but through a distant common ancestor. The picture is similar for Families 4, 5, 6, and 7, UK families of Pakistani origin. In contrast, Families 8 and 9 from Oman share an identical haplotype across the region tested. These findings, therefore, suggest that MMP2O c. $710 \mathrm{C}>\mathrm{A}$ represents a relatively recent founder mutation in the Omani population, while c.954-2A>T and c.625G $>C$ result from older founder mutations in the Caucasian European and Pakistani populations.

In addition to the novel p.(Glu209GIn) and p.(Ser237Tyr) variants, we also performed MD for known variants p.(Leu189Pro) and p.(Thr130lle) to see if the simulations could provide equivalent structural insight into the loss of function. Other variants within the active site of MMP20 that change the ability of zinc ions to form coordination bonds were not modeled because this will either lead to substantial structural distortion (as seen with MD in the absence of calcium or zinc ions), or destabilize the key zinc metal center essential for catalysis.

For p.(Leu189Pro), while there is only a modest increase in RMSD relative to the WT, simulation of the behavior of the bound ligand in the WT and variant shows that replacing bulky lysine with more compact proline changes the shape of the active site. This leads to substantial structural rearrangement of the ligand, which is likely to affect substrate recognition and catalytic activity of the protease against natural substrates.

The only pathogenic missense variant modeled that did not show a significantly larger RMSD than the WT was p.(Thr130lle). While minor changes in hydrogen bonding interactions were detected (Table S8), no substantial changes in overall protein structure or perturbations to interatomic interactions with the ligand or metal ions were observed. Consequently, our MD simulations do not provide a structural mechanism for why this variant should be pathogenic. However, p.(Thr130lle) is located at the edge of the catalytic domain, close to regions of the protein that are absent from the available structure and which, therefore, do not feature in these simulations.

The 14 MMP20 variants previously reported to cause $\mathrm{Al}$ are shown in Figure 3, together with those newly described herein, indicating their location in the gene and protein. Previously reported pathogenic variants consisted of seven missense variants, two premature stop codons, two frameshifts, and two putative splice variants. Here, we report a further three missense and one splice variants, consistent with and extending the spectrum of mutations in MMP2O causing autosomal recessive Al. The three splice variants have not been verified beyond in silico prediction and therefore remain likely but unproven. These 17 variants, and in particular, the missense variants, cluster primarily in or near to the zinc-dependent peptidase domain (Figure 3). Our analyses suggest that pathogenic mutations in this domain alter protein stability, while others (Y. J. Kim et al., 2017) have shown that variants in the catalytic domain can lead to a reduction or complete loss of enzymatic function. The combination of recessive inheritance, missense variants that reduce or abolish function, and the lack of significant difference between phenotypes associated with missense and nonsense variants, all point to a loss-of-function phenotype, where lack of functional MMP20 gives rise to a consistent hypomaturation $\mathrm{Al}$ phenotype.

In summary, we have identified 10 families segregating Al due to four novel and one known mutations in MMP20, and reviewed previously reported mutations, raising the total number of Al-causing MMP20 variants to 18 . This expands the spectrum of MMP20 variants implicated in $\mathrm{Al}$ and confirms the association with a hypomaturation phenotype. Haplotype analysis suggests that the c.710C>A, p.(Ser237Tyr); c.625G >C, p.(Glu209GIn); and c.954$2 A>T$, p.(Ile319Phefs*19) variants may be relatively common founder mutations in the Omani, Pakistani, and Caucasian populations, respectively. Biochemical modeling and MD analyses show that missense mutations in the catalytic domain alter protein stability, suggesting that this form of $\mathrm{Al}$ is the result of the loss-offunction of MMP20.

\section{ACKNOWLEDGMENTS}

The authors thank the families involved in this study. This study was supported by the Welcome Trust (Grant: 093113). Georgios Nikolopoulos was funded by a PhD scholarship funded by Leeds Dental School, Faculty of Medicine and Health, University of Leeds. CELS was funded by a Wellcome Trust Institutional Strategic Support Fund award. Funding for Open Access publication charges for this article was provided by the Wellcome Trust. This study was undertaken on ARC3, part of the High-Performance Computing Facilities at the University of Leeds, UK.

\section{CONFLICT OF INTERESTS}

The authors declare that there are no conflict of interests.

\section{DATA AVAILABILITY STATEMENT}

The data that support the findings of this study are openly available in ClinVar at https://www.ncbi.nlm.nih.gov/clinvar/, accession numbers: SCV001338799-SCV001338802 and in the Al Leiden Open Variation Database (LOVD) at http://dna2.leeds.ac.uk/LOVD/ with reference numbers: 0000000313-0000000317. 


\section{WEB RESOURCES}

- The AI Leiden Open Variation Database (LOVD; http://dna2.leeds. ac.uk/LOVD/).

- ClinVar (https://www.ncbi.nlm.nih.gov/clinvar/).

- The Genome Aggregation Database (gnomAD; http://gnomad. broadinstitute.org).

- Combined Annotation-Dependent Depletion (CADD v1.3; https:// cadd.gs.washington.edu).

- MutPred2 (http://mutpred.mutdb.org/index.html).

- Human Splicing Finder v3.1 (http://umd.be/HSF3/HSF.shtml).

\section{ORCID}

Georgios Nikolopoulos (D) http://orcid.org/0000-0003-3166-8372

Claire E. L. Smith (D) http://orcid.org/0000-0001-8320-5105

James A. Poulter (D) http://orcid.org/0000-0003-2048-5693

Gina Murillo (D) http://orcid.org/0000-0003-2976-6301

Peter F. Day (D) http://orcid.org/0000-0001-9711-9638

Sarah A. Harris (D) http://orcid.org/0000-0002-2812-1651

Mary J. O'Connell (D) http://orcid.org/0000-0002-1877-1001

Chris F. Inglehearn (D) https://orcid.org/0000-0002-5143-2562

Alan J. Mighell (D) https://orcid.org/0000-0002-9624-6923

\section{REFERENCES}

Andreini, C., Banci, L., Bertini, I., Luchinat, C., \& Rosato, A. (2004). Bioinformatic comparison of structures and homology-models of matrix metalloproteinases. Journal of Proteome Research, 3(1), 21-31. https://doi.org/10.1021/pr0340476

Arendt, Y., Banci, L., Bertini, I., Cantini, F., Cozzi, R., Del Conte, R., \& Gonnelli, L. (2007). Catalytic domain of MMP20 (Enamelysin)-The NMR structure of a new matrix metalloproteinase. FEBS Letters, 581(24), 4723-4726. https://doi.org/10.1016/j.febslet.2007.08.069

Bartlett, J. D. (2013). Dental enamel development: Proteinases and their enamel matrix substrates. ISRN Dentistry, 2013, 1-24. https://doi. org/10.1155/2013/684607

Case, D. A., Ben-Shalom, I. Y., Brozell, S. R., Cerutti, D. S., Cheatham, T. E., III, Cruzeiro, V. W. D., Darden, T. A., Duke, R. E., Ghoreishi, D., \& Gilson, M. K. (2018). AMBER 2018. University of California, San Francisco: http://ambermd.org/CiteAmber.php

Choi, Y., Sims, G. E., Murphy, S., Miller, J. R., \& Chan, A. P. (2012). Predicting the functional effect of amino acid substitutions and indels. PLOS One, 7(10), e46688. https://doi.org/10.1371/journal. pone.0046688

Dubail, J., Huber, C., Chantepie, S., Sonntag, S., Tüysüz, B., Mihci, E., Gordon, C. T., Steichen-Gersdorf, E., Amiel, J., Nur, B., Stolte-Dijkstra, I., van Eerde, A. M., van Gassen, K. L., Breugem, C. C., Stegmann, A., Lekszas, C., Maroofian, R., Karimiani, E. G., Bruneel, A., ... Cormier-Daire, V. (2018). SLC10A7 mutations cause a skeletal dysplasia with amelogenesis imperfecta mediated by GAG biosynthesis defects. Nature Communications, 9(1), 3087. https:// doi.org/10.1038/s41467-018-05191-8

Gadhia, K., McDonald, S., Arkutu, N., \& Malik, K. (2012). Amelogenesis imperfecta: An introduction. British Dental Journal, 212(8), 377-379. https://doi.org/10.1038/sj.bdj.2012.314

Gasse, B., Prasad, M., Delgado, S., Huckert, M., Kawczynski, M., GarretBernardin, A., Lopez-Cazaux, S., Bailleul-Forestier, I., Manière, M. C., Stoetzel, C., Bloch-Zupan, A., \& Sire, J. Y. (2017). Evolutionary analysis predicts sensitive positions of MMP20 and validates newlyand previously-identified MMP20 mutations causing amelogenesis imperfecta. Frontiers in Physiology, 8, 398. https://doi.org/10.3389/ fphys.2017.00398

Guan, X., \& Bartlett, J. D. (2013). MMP20 modulates cadherin expression in ameloblasts as enamel develops. Journal of Dental Research, 92(12), 1123-1128. https://doi.org/10.1177/0022034513506581

Kim, J. W., Simmer, J. P., Hart, T. C., Hart, P. S., Ramaswami, M. D., Bartlett, J. D., \& Hu, J. C. C. (2005). MMP-20 mutation in autosomal recessive pigmented hypomaturation amelogenesis imperfecta. Journal of Medical Genetics, 42(3), 271-275. https://doi.org/10. 1136/jmg.2004.024505

Kim, J. W., Zhang, H., Seymen, F., Koruyucu, M., Hu, Y., Kang, J., Kim, Y. J., Ikeda, A., Kasimoglu, Y., Bayram, M., Zhang, C., Kawasaki, K., Bartlett, J. D., Saunders, T. L., Simmer, J. P., \& Hu, J. C. (2019). Mutations in RELT cause autosomal recessive amelogenesis imperfecta. Clinical Genetics, 95(3), 375-383. https://doi.org/10. 1111/cge.13487

Kim, Y. J., Kang, J., Seymen, F., Koruyucu, M., Gencay, K., Shin, T. J., Hyun, H. K., Lee, Z. H., Hu, J. C. C., Simmer, J. P., \& Kim, J. W. (2017). Analyses of MMP20 missense mutations in two families with hypomaturation amelogenesis imperfecta. Frontiers in Physiology, 8, 229. https://doi.org/10.3389/fphys.2017.00229

Llano, E., Pendás, A. M., Knäuper, V., Sorsa, T., Salo, T., Salido, E., Murphy, G., Simmer, J. P., Bartlett, J. D., \& López-Otín, C. (1997). Identification and structural and functional characterization of human enamelysin (MMP-20). Biochemistry, 36(49), 15101-15108. https://doi.org/10.1021/bi972120y

Nikolopoulos, G., Smith, C. E. L., Brookes, S. J., El-Asrag, M. E., Brown, C. J., Patel, A., Murillo, G., O'Connell, M. J., Inglehearn, C. F., \& Mighell, A. J. (2020). New missense variants in RELT causing hypomineralised amelogenesis imperfecta. Clinical Genetics, 97(5), 688-695. https://doi.org/10.1111/cge.13721

Pandurangan, A. P., Ochoa-Montaño, B., Ascher, D. B., \& Blundell, T. L. (2017). SDM: A server for predicting effects of mutations on protein stability. Nucleic Acids Research, 45(W1), W229-W235. https://doi. org/10.1093/nar/gkx439

Pettersen, E. F., Goddard, T. D., Huang, C. C., Couch, G. S., Greenblatt, D. M., Meng, E. C., \& Ferrin, T. E. (2004). UCSF Chimera-A visualization system for exploratory research and analysis. Journal of Computational Chemistry, 25(13), 1605-1612. https://doi.org/10.1002/ jcc.20084

Ponzoni, L., Peñaherrera, D. A., Oltvai, Z. N., \& Bahar, I. (2020). Rhapsody: Predicting the pathogenicity of human missense variants. Bioinformatics, 36, 3084-3092. https://doi.org/10.1093/bioinformatics/btaa127

Prasad, M. K., Geoffroy, V., Vicaire, S., Jost, B., Dumas, M., Le Gras, S., Switala, M., Gasse, B., Laugel-Haushalter, V., Paschaki, M., Leheup, B., Droz, D., Dalstein, A., Loing, A., Grollemund, B., Muller-Bolla, M., Lopez-Cazaux, S., Minoux, M., Jung, S., ... Bloch-Zupan, A. (2015). A targeted next-generation sequencing assay for the molecular diagnosis of genetic disorders with orodental involvement. Journal of Medical Genetics, 53(2), 98-110. https://doi.org/10.1136/ jmedgenet-2015-103302

Rentzsch, P., Witten, D., Cooper, G. M., Shendure, J., \& Kircher, M. (2019). CADD: Predicting the deleteriousness of variants throughout the human genome. Nucleic Acids Research, 47(D1), D886-D894. https:// doi.org/10.1093/nar/gky1016

Seymen, F., Park, J. C., Lee, K. E., Lee, H. K., Lee, D. S., Koruyucu, M., Gencay, K., Bayram, M., Tuna, E. B., Lee, Z. H., Kim, Y. J., \& Kim, J. W. (2015). Novel MMP20 and KLK4 mutations in amelogenesis imperfecta. Journal of Dental Research, 94(8), 1063-1069. https:// doi.org/10.1177/0022034515590569

Sim, N. L., Kumar, P., Hu, J., Henikoff, S., Schneider, G., \& Ng, P. C. (2012). SIFT web server: Predicting effects of amino acid substitutions on proteins. Nucleic Acids Research, 40(W1), W452-W457. https://doi. org/10.1093/nar/gks539 
Simmer, J. P., \& Hu, J. C. C. (2002). Expression, structure, and function of enamel proteinases. Connective Tissue Research, 43(2-3), 441-449. https://doi.org/10.1080/713713530

Smith, C. E. (1998). Cellular and chemical events during enamel maturation. Critical Reviews in Oral Biology and Medicine, 9(2), 128-161. https://doi.org/10.1177/10454411980090020101

Smith, C. E. L., Poulter, J. A., Antanaviciute, A., Kirkham, J., Brookes, S. J., Inglehearn, C. F., \& Mighell, A. J. (2017). Amelogenesis imperfecta; genes, proteins, and pathways. Frontiers in Physiology, 8, 435. https:// doi.org/10.3389/fphys.2017.00435

Smith, C. E. L., Poulter, J. A., Brookes, S. J., Murillo, G., Silva, S., Brown, C. J., Patel, A., Hussain, H., Kirkham, J., Inglehearn, C. F., \& Mighell, A. J. (2019). Phenotype and variant spectrum in the LAMB3 form of amelogenesis imperfecta. Journal of Dental Research, 98(6), 698-704. https://doi.org/10.1177/0022034 519835205

Smith, C. E. L., Whitehouse, L. L. E., Poulter, J. A., Hewitt, L. W., Nadat, F., Jackson, B. R., Manfield, I. W., Edwards, T. A., Rodd, H. D., Inglehearn, C. F., \& Mighell, A. J. (2020). A missense variant in specificity protein 6 (SP6) is associated with amelogenesis imperfecta. Human Molecular Genetics, 29, 1417-1425. https://doi. org/10.1093/hmg/ddaa041

Wright, J. T., Carrion, I. A., \& Morris, C. (2015). The molecular basis of hereditary enamel defects in humans. Journal of Dental Research, 94(1), 52-61. https://doi.org/10.1177/0022034514 556708
Wright, J. T., Torain, M., Long, K., Seow, K., Crawford, P., Aldred, M. J., Hart, P. S., \& Hart, T. C. (2011). Amelogenesis imperfecta: Genotypephenotype studies in 71 families. Cells Tissues Organs, 194(2-4), 279-283. https://doi.org/10.1159/000324339

Yamakoshi, Y., Simmer, J. P., Bartlett, J. D., Karakida, T., \& Oida, S. (2013). MMP2O and KLK4 activation and inactivation interactions in vitro. Archives of Oral Biology, 58(11), 1569-1577. https://doi.org/10. 1016/j.archoralbio.2013.08.005

\section{SUPPORTING INFORMATION}

Additional Supporting Information may be found online in the supporting information tab for this article.

How to cite this article: Nikolopoulos, G., Smith, C. E. L., Poulter, J. A., Murillo, G., Silva, S., Lamb, T., Berry, I. R., Brown, C. J., Day, P. F., Soldani, F., Al-Bahlani, S., Harris, S. A., O'Connell, M. J., Inglehearn, C. F., \& Mighell, A. J. (2021). Spectrum of pathogenic variants and founder effects in amelogenesis imperfecta associated with MMP20. Human Mutation, 42, 567-576. https://doi.org/10.1002/humu.24187 